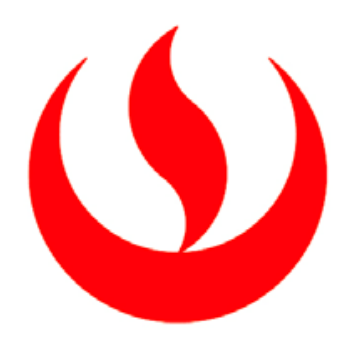

Universidad Peruana de Ciencias Aplicadas

FACULTAD DE CIENCIAS DE LA SALUD

CARRERA DE NUTRICIÓN Y DIETÉTICA

\title{
Traducción y validación de la versión al español del cuestionario orto-15 para la evaluación de ortorexia
}

Tesis para optar el título profesional de:

Licenciada en Nutrición y Dietética

\author{
Autores \\ Vanessa Gabriela Cavero Amayo \\ Cynthia Lodwig León \\ Gabriela Lulli Rodriguez-Larraín
}

Asesores de tesis

Romina Tejada Caminiti

Jorge Maguiña Quispe

Percy Mayta-Tristán

Lima, 2017 
Dedicatoria

Dedicado con cariño a nuestros padres y familia, quienes permanentemente nos apoyaron con espíritu alentador, contribuyendo a lograr los objetivos propuestos. 


\section{Agradecimientos}

Agradecemos nuestros asesores por el soporte y la participación en la elaboración de la presente investigación. 


\section{ÍNDICE}

ÍNDICE

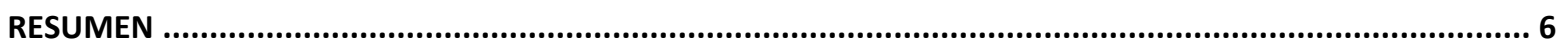

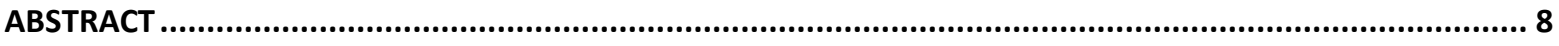

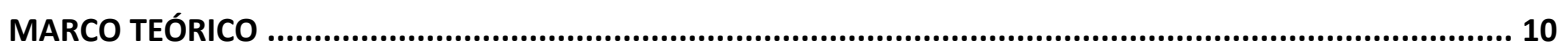

OBJETIVOS

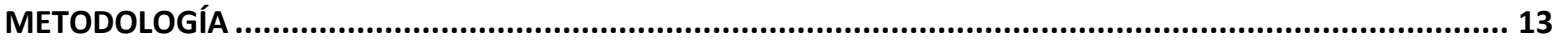

RESULTADOS

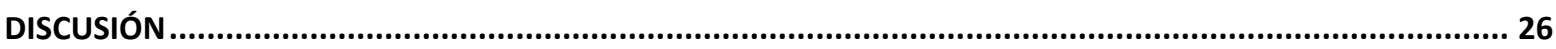

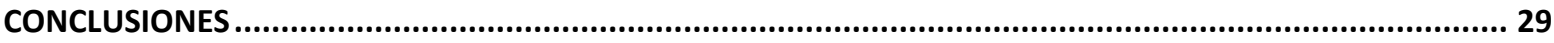

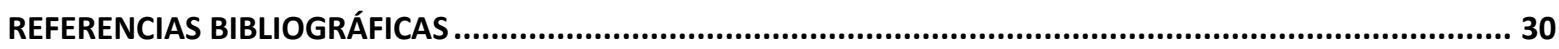

ANEXOS 


\section{RESUMEN}

\section{Introducción}

La ortorexia nerviosa $(\mathrm{ON})$ es un cuadro de obsesión por la comida saludable. El ORTO-15 es el cuestionario, en idioma italiano, que se utiliza para medir la tendencia a la ON. También se han generado versiones distintas como el ORTO-11 y ORTO-9. Es importante resaltar que no se ha encontrado una versión traducida ni validada del cuestionario ORTO15 al idioma español.

\section{Objetivos}

Traducir el ORTO-15 del idioma español y validarlo en una población de jóvenes universitarias mujeres.

\section{Métodos}

Se realizó un estudio de validación del cuestionario ORTO-15 en tres fases: La fase (1) traducción, (3) fase de adaptación cultural y fase (3) de validación aplicada, con el cuestionario final desarrollado. 


\section{Resultados}

El ORTO-15 presentó los siguientes índices de ajuste CFI $=0,783$; TLI $=0,738$; RMSEA 0,072; $\chi^{2}=466,38$ y un alfa de Cronbach $=0,73$. El modelo 2 del ORTO 11 presentó un CFI $=0,853 ;$ TLI $=0,816$; RMSEA 0,074; $\chi 2=102,9$ y un alfa de Cronbach $=0,74$. Finalmente el modelo 3 del ORTO 9 presentó (CFI = 0,752; TLI =0,669; RMSEA 0.096; $\chi 2=91,27$ y un alfa de Cronbach $=0,64)$. El modelo 2, fue el que mejor se ajusta a nuestros datos y se adapta a nuestra realidad.

\section{Conclusión}

El mejor cuestionario para evaluar la tendencia a la $\mathrm{ON}$ en la población estudiada es el ORTO 11, el cual presentó mejores propiedades psicométricas en comparación con el ORTO 9 y el ORTO 15.

\section{Keywords}

Ortorexia Nerviosa, Conducta Alimentaria, Estudios de Validación, Mujeres, Perú. 


\section{ABSTRACT}

TRANSLATION AND VALIDATION OF THE SPANISH VERSION QUESTIONNAIRE ORTHO-15 FOR THE EVALUATION OF ORTHOREXIA.

\section{Introduction}

Orthorexia Nervosa (ON) is an obsession for healthy eating. There is a diagnostic questionnaire, named ORTHO-15, developed in Italian language to measure the orthorexia tendency. There are also similar questionnaires known as the ORTHO-11 and the ORTHO-9, which are adaptations from the ORTHO-15. It is important to mention that there is no known translation of the questionnaire ORTHO-15 to Spanish language with its corresponding validation.

\section{Aims}

Translate the ORTHO-15 into Spanish and validate it into our target population among women in undergraduate college.

\section{Methods}

A validation study of the questionnaire ORTHO-15 was conducted in three phases:

Phase (1) was the translation, in step (2) cultural adaptation and expert judgment, finally in the phase (3) we applied the validation, developed with the final questionnaire.

\section{Findings}

The ORTHO-15 presented the following fit indices CFI $=0,783$; TLI $=0,738$; RMSEA 0,072; $\chi^{2}=466,38$ and Cronbach's alpha $=0,73$. The model 2 of ORTHO 11 from the adapted version of Hungary presented CFI $=0,853$; TLI $=0,816$; RMSEA $0.074 ; \chi 2=102$, 9 and Cronbach's alpha = 0, 74. Finally, the model 3 of ORTHO 9 from Alemania presented $\mathrm{CFI}=0,752 ; \mathrm{TLI}=0,669 ; \mathrm{RMSEA} 0.096 ; \chi 2=91,27$ and Cronbach's alpha $=0,64$. Of the 
three models reported, the model 2 was the one that best fitted our data and best adapted to our reality.

\section{Conclusion}

The best questionnaire to assess the tendency to $\mathrm{ON}$ in the target population is the ORTHO11 which presented better psychometric properties compared with ORTHO-9 and ORTHO15. 


\section{MARCO TEÓRICO}

En 1997 el médico norteamericano Steven Bratman, inició el estudio sobre la ortorexia nerviosa $(\mathrm{ON})$, la cual está definida como un cuadro obsesivo por la comida saludable. (1) Las personas con tendencia de ON presentan ciertas características en su comportamiento que puede representar una limitación y afectar su calidad de vida, como la perfección distorsionada por una alimentación saludable, la cual altera la forma en que perciben a las demás personas. Además, sufren de aislamiento social, ya que discriminan a las personas que no comparten su misma ideología de alimentación. (2) Asimismo, tienen un control y preocupación excesiva sobre la preparación de los alimentos y los tipos de utensilios de cocina que utilizan para ello. (3) Son exigentes en cuanto a consumir alimentos sin colorantes, preservantes, pesticidas, alimentos genéticamente modificados, grasas trans o contenidos excesivos de sal, azúcar y otros componentes que ellos consideren que podrían ocasionan daño a la salud. (1)

Las personas con tendencia a la $\mathrm{ON}$ se respaldan en la creencia de que están "comiendo sano". Esta obsesión aún no está reconocida como una enfermedad dentro del Manual Diagnóstico y Estadístico de Trastornos Mentales (DSM-V) de la Asociación Americana de Psiquiatría. (4) Debido a que es un cuadro estudiado recientemente, (5) y aún no existe suficiente evidencia científica que lo califique como un trastorno alimentario.

Existen dos instrumentos principales para evaluar la tendencia a desarrollar ON; sin embargo, éstos no la diagnostican. El primero fue creado por Bratman, quien elaboró un cuestionario de diez preguntas con respuestas dicotómicas, que al ser basado únicamente en su experiencia clínica y no estar validado correctamente fue modificado. (1) Dicho instrumento fue modificado por Donini, quien mantuvo seis preguntas $(1,3,7,8,9$, y 10) y agregó las restantes, cambiando las respuestas a una escala Likert con el fin de obtener un cuestionario más específico basado en las características principales de la ON. Además, realizó la validación del instrumento, el cual es conocido como ORTO 15, y ha sido el más utilizado en diversos estudios. $(3,6,7)$. Sin embargo, en Latinoamérica no se ha encontrado 
estudios que hayan realizado una traducción y validación del cuestionario ORTO-15 al idioma español, ya que sólo tradujeron el instrumento del inglés al español con fines descriptivos más no psicométricos.

El cuestionario elaborado por Bratman, se basa principalmente en la experiencia clínica y no ha sido validado. Asimismo, a partir del ORTO-15 se han generado nuevas versiones con distintas estructuras como ORTO-11 elaborado en Turquía y Hungría, y ORTO- 9 en Alemania. Estas nuevas versiones omiten algunos ítems para obtener una mejor consistencia interna, siendo el ORTO-11 de Hungría el que mejor se adapta, con un alfa de Cronbach de 0,82. Asimismo, el ORTO-15 no reporta un alfa de Cronbach. Estas nuevas versiones fueron creadas con el fin de mejorar la validez del instrumento. $(3,8-10)$

Es importante tener disponible un instrumento que ayude a los profesionales de la salud a detectar el riesgo de presentar características ortoréxicas y de esta manera poder abordar la tendencia a la misma con éxito. (11) 


\section{OBJETIVOS}

\section{General}

Traducir el ORTO-15 al español y validarlo en una población de jóvenes universitarias mujeres.

\section{Específicos}

Traducir el cuestionario ORTO-15 del idioma original italiano al idioma español.

Adaptar el cuestionario ORTO-15 para la población peruana.

Analizar la validez de constructo, validez convergente y fiabilidad de la versión al español del ORTO-15. 


\section{METODOLOGÍA}

Se realizó un estudio de validación en tres fases: (a) traducción, (b) adaptación cultural y (c) validación. El estudio fue revisado y aprobado por el Comité de Ética del Hospital Nacional Docente Madre- Niño San Bartolomé (CODIGO 02777-15) y por la Escuela de Nutrición y Dietética de la Universidad Peruana de Ciencias Aplicadas en Lima, Perú. Todos los participantes incluidos firmaron un consentimiento informado escrito.

\section{Descripción del instrumento}

El ORTO-15 fue desarrollado en Italia por Donini, mide la tendencia de ON, tiene 15 ítems con respuestas de tipo Likert de cuatro opciones que van de siempre a nunca, analizando aspectos vinculados con hábitos alimentarios, prácticas culinarias y actitudes frente a su alimentación. Tiene ítems con puntuación reversa $(2 / 5 / 8 / 9: 4,3,2,1)$, ítems con puntación regular $(3 / 4 / 6 / 7 / 10 / 11 / 12 / 14 / 15: 1,2,3,4)$ e ítems con una puntuación alterna $(1 / 13: 2,4,3,1)$. En su versión original se agrupan en tres dominios: área clínica (3/7/8/9/15), cognitivaracional $(1 / 5 / 6 / 11 / 12 / 14)$ y emocional $(2 / 4 / 10 / 13)$ de la persona. A mayor puntuación implica menor riesgo de ortorexia; asimismo, el punto de corte sugerido es menor a 40. En las traducciones realizadas a otros idiomas, se han encontrado versiones con menor número de ítems y con un solo dominio, ORTO-9 en alemán, ORTO-11 en húngaro y turco. (8-10)

\section{Traducción}

Se realizó el proceso en cinco pasos según las recomendaciones internacionales (12). (a) Traducción directa, se usó la versión original en italiano solicitada al autor de la escala a diferencia de estudios previos que usaron la versión en inglés disponible en el artículo donde se publicó la validación del ORTO-15 (3). Dos traductores de lengua materna español, uno de ellos nutricionista, generaron traducciones independientes. (b) Síntesis en español, los investigadores generaron una sola versión a partir de las traducciones recibidas y está versión fue aprobada por los traductores. (c y d) Traducción inversa y síntesis de traducción 
inversa (español a italiano), similar a los pasos a y b, pero esta vez los traductores tenían como lengua materna el italiano. (e) Equivalencia semántica, la versión retraducida al italiano fue enviada al autor que creó el instrumento original para su revisión y aprobación.

\section{Adaptación cultural}

Se realizó en dos fases, (a) juicio de expertos, y (b) prueba piloto. Se trabajó con un panel de seis expertos (dos nutricionistas, un lingüista, dos investigadores metodólogos y un psicólogo) que siguiendo la metodología Delphi evaluaron la claridad, coherencia, relevancia y adaptación cultural a la realidad peruana con puntajes del 0 a 4 , donde adicionalmente apuntaban comentarios o sugerencias del cambio de equivalencia idiomática del cuestionario traducido. (13) La versión final obtenida se probó en un piloto con 20 estudiantes entre 18 y 30 años de diversas carreras no pertenecientes a las carreras de ciencias de la salud donde se evaluó la comprensión de cada pregunta y si entendían los términos incluidos.

\section{Validación}

Se evaluó la validez de constructo, validez de contenido, convergente y fiabilidad con diferentes poblaciones.

Validez de contenido. Se evaluó con el juicio de expertos mencionado en la adaptación cultural.

Validez convergente. Se evaluó con 35 estudiantes de medicina mujeres de la UPC a las cuales se les aplicó la versión en español del ORTO-15 y la versión validada al español del EAT-26 (14) y se evaluó su correlación entre los puntajes totales, tal como se hizo en un estudio previo (6). 


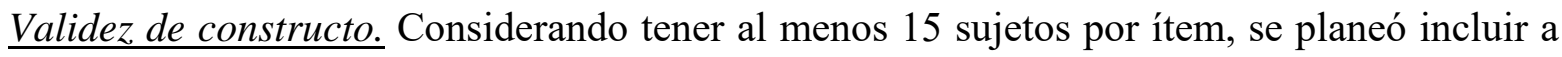
más de 225 personas. (15) Se invitó a participar a estudiantes mujeres entre 18 y 30 años de las carreras de Nutrición y Dietética y Diseño de Interiores matriculadas en la Universidad Peruana de Ciencias Aplicadas (UPC) en el primer semestre del año 2015, seleccionadas por muestreo no probabilístico, por conveniencia. Con estos datos se evaluó, con el análisis factorial confirmatorio, si los datos ajustaban según el modelo inicial de Donini.

Fiabilidad. Con la primera muestra $(\mathrm{n}=256)$ se evaluó la consistencia interna con el alfa de Cronbach. Para medir la estabilidad se realizó un test-retest con la segunda muestra $(n=35)$ a las cuales se le aplicó nuevamente el ORTO-15 una semana después para evaluar la correlación intraclase y el cambio de puntaje a nivel de ítems y de la escala global. Tanto para los valores de alfa de Cronbach como de correlación intraclase se consideró valores iguales o superiores a 0,7 como adecuados.

\section{Asociación entre el puntaje del ORTO-15 con otras variables}

En la población trabajada para la validez de constructo, se aplicó el cuestionario en forma anónima, el cual incluía el ORTO 15 y preguntas adicionales sobre datos sociodemográficos (edad, género y pensión universitaria), antecedentes patológicos del encuestado y de familiares en primer grado, así como realización de actividad física. Finalmente, fueron pesados y tallados siguiendo la guía técnica de valoración nutricional de la persona adulta del Ministerio de Salud del Perú (16).

\section{Análisis de datos}

Los datos fueron ingresados por doble digitación en el programa Excel 2010, luego del control de calidad fueron exportados a STATA v14.0 para el análisis estadístico. Las variables categóricas se expresaron en frecuencias relativas y las numéricas en media y desviación estándar. 
Debido a la variedad e inconsistencia en la validez de los instrumentos que miden $\mathrm{ON}$, evaluamos los constructos previamente reportados por Donini de tres dimensiones y 15 ítems (modelo 1) (3), de Varga et al de una dimensión y 11 ítems (modelo 2) (9) y el de Missbach et al de una dimensión y nueve ítems (modelo 3). (10) Para ello empleamos un análisis factorial confirmatorio (AFC) usando modelos de ecuaciones estructurales para su comparación y evaluación a través de los índices de bondad de ajuste, tales como CFI: índice de ajuste comparativo, TLI: índice Tucker-Lewis, RMSEA: aproximación del error de los mínimos cuadrados, alfa de Cronbach y el chi-cuadrado likelihood. Donde un CFI, TLI y el alfa de Cronbach cercanos a uno y un RMSEA cercano a cero indica un buen modelo de ajuste.

Se evaluó la validez convergente con la correlación de Pearson entre los puntajes del ORTO al español y el EAT-26. Para la consistencia interna se calculó el alfa de Cronbach; para la estabilidad en el test-retest se calculó el coeficiente de correlación intraclase, el cambio de puntaje total y de cada uno de los ítems se evaluó con la prueba de los signos de Wilcoxon. Finalmente, se evaluó la asociación entre el puntaje global de la versión al español del ORTO-11 con la edad usando la prueba de correlación de Pearson y las pruebas de $\mathrm{T}$ de student y el análisis de varianzas de una vía (ANOVA) para las diferencias de promedios en las variables categóricas. Se consideró un $\mathrm{p}<0,05$ como significativo. 


\section{RESULTADOS}

\section{Traducción y adaptación cultural}

Se realizó este proceso según la metodología descrita, la versión retraducida al italiano fue enviada al autor original quien corroboró la equivalencia semántica del instrumento. La versión al español obtenida fue adaptada durante el juicio de expertos. Las nutricionistas realizaron cambios en las preguntas que hacían mención a confusión al momento de elegir un producto alimentario, cambios como “ ¿El sabor es lo más importante que la calidad a la hora de comer?”, “¿Presentas rechazo o negación hacia un grupo de alimentos?”, si “¿Tiene sentimiento de culpabilidad cuando realizas una trasgresión alimentaria?”, y si “¿Considera presencia de comida no sana en mercados?”. Los metodólogos modificaron preguntas como “¿Consideras que la comida sana modifica tu estilo de vida?”, “En los últimos tres meses pensar en comida es una preocupación para ti?”, “Cuando consumes comidas no sanas lo haces solo o acompañado y el hecho de pensar en comida es una preocupación por más de tres horas al día”. La versión final en español a evaluar se muestra en la (ver tabla 1). 
Tabla 1. ORTO 15 versión original en italiano y en español.

\section{ORTO 15 original (Italiano)}

1. Quando mangia fa attenzione alle calorie degli alimenti?

2. Quando entra in un negozio di prodotti alimentari, è confuso?

3. Negli ultimi 3 mesi, il pensiero del cibo è stato per lei una preoccupazione?

4. Le sue scelte alimentari sono condizionate dalla preoccupazione per il suo stato di salute?

5. 11 sappore è la più importante delle qualità di cui lei tiene conto nel giudicare un cibo?

6. E' disposto a spendere di più per avere un cibo sano?

7. 11 pensiero del cibo sano la preoccupa per piú di tre ore al giorno?

8. Si concede qualche trasgressione alimentare?

9. Ritiene che il tono dell'umore incida sul suo comportamento alimentare?

10. Ritiene che la convinzione di alimentarsi con cibi sani aumenti la sua autostima?

11. Ritiene che il consumo di cibi sani modifichi il suo stile di vita (frequenza ritorante, amici,...)?

12. Ritiene che consumare cibi sani possa migliorare il suo aspetto fisico?

13. Si sente in colpa quando trasgredisce?

14. Ritiene che in commercio siano presenti anche cibi non sani?

15. Attualmente quando consuma i pasti, lofa da solo?
ORTO 15 Traducido (Español)

1. ¿Piensas en cuántas calorías consumes cuando comes?

2. ¿En una tienda de productos alimentarias es difícil para ti elegir alimentos?

3. ¿En los últimos tres meses pensar en comida ha sido una preocupación para ti?

4. ¿Lo que eliges para comer está condicionado por la preocupación por tu estado de salud?

5. ¿El sabor es la cualidad más importante que tiene en cuenta a la hora de juzgar un alimento?*

6. ¿Estás dispuesta gastar más dinero por una alimentación saludable?*

7. ¿El pensar en comida sana ha sido una preocupación por más de 3 horas?

8. ¿Tienes algún rechazo o negación a un alimento o grupo de alimentos?*

9. ¿Consideras que el estado de ánimo afecta cómo y cuánto decido comer?

10. ¿Crees que sea verdad que la comida sana te hace sentir bien contigo misma?

11. ¿Consideras que el consumo de comida sana modifica tu comportamiento con tus amistades y/o familiares

12. ¿Consideras que consumir comida sana puede mejorar tu aspecto físico?

13. ¿Te sientes culpable cuando consumes alimentos poco saludables?

14. ¿Consideras que el mercado actual de productos alimentarios tiene en su mayoría productos no sanos?*

15. ¿Actualmente cuando consumes tus alimentos lo haces solo?

*Preguntas omitidas que generan el ORTO11. Puntuación de ítems: 3/4/7/10/11/12/15: 1,2,3,4; 2/9: 4,3,2,1; 1/13: 2,4,3,1. 


\section{Validez convergente}

Se evaluó la correlación entre el puntaje total obtenido en el ORTO-11 con el EAT-26 y se encontró un coeficiente de correlación de Pearson de $-0,44(\mathrm{p}<0,001)$.

\section{Validez de constructo}

En primer lugar evaluamos el modelo original de tres dominios del ORTO-15. Nosotros evaluamos tres diferentes modelos con nuestros datos. El modelo 1 (ORTO-15), basado en la versión original de Donini, el modelo 2 (ORTO-11) basado en la versión húngara adaptada de Vargas et al y finalmente el modelo 3 (ORTO-9) basado en la versión alemana adaptada de Missbach et al (ver figura 1). 


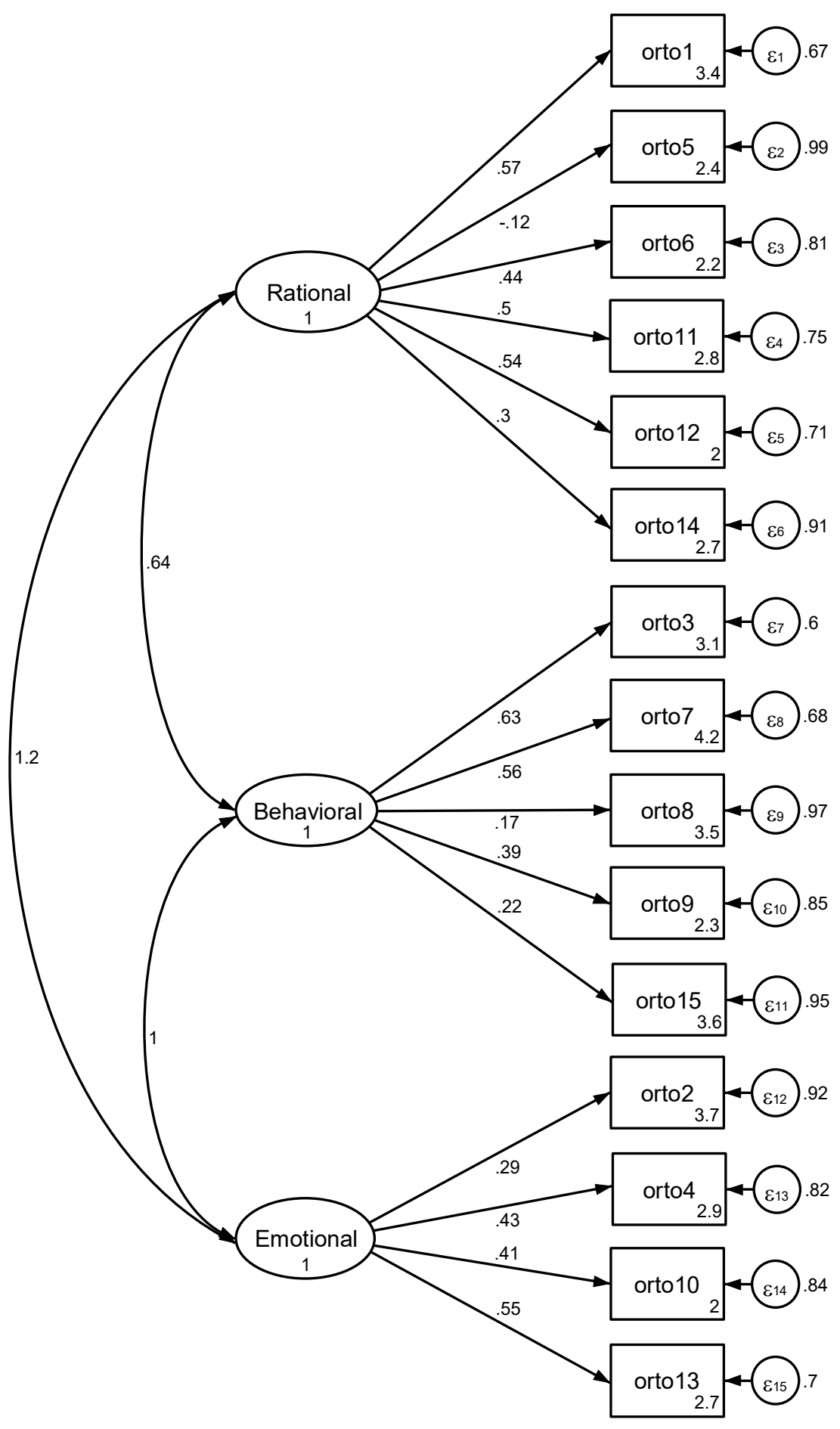

Figura 1. Mejor modelo final de ajuste ORTO 15 con tres dimensiones. 
El modelo 1 presentó los siguientes índices de ajuste $\mathrm{CFI}=0,783$; TLI $=0,738$; RMSEA 0.072; $\chi 2=466,38$ y un alfa de Cronbach $=0,73$. El modelo $2, \mathrm{CFI}=0,853$; TLI $=0,816$; RMSEA 0.074; $\chi 2=102,9$ y un alfa de Cronbach $=0,74$. Finalmente, el modelo $3, \mathrm{CFI}=$ 0,752; TLI =0,669; RMSEA 0,096; $\chi 2=91,27$ y un alfa de Cronbach $=0,64$ (ver tabla 2). De los tres modelos reportados, a pesar que el modelo 2 presento índices de ajuste bajos, este fue el que mejor se ajusta a nuestros datos y se adapta a la realidad estudiada. Se observa también el factor de carga del ORTO-15 y ORTO-11 (ver figura 2 y tabla 3). 
Tabla 2. Comparación de los modelos de ajuste post análisis factorial confirmatorio de los cuestionarios ORTO 15, ORTO 11 y ORTO 9 en las versiones en húngaro, alemán y español.

\begin{tabular}{|c|c|c|c|c|c|c|c|}
\hline \multirow[b]{2}{*}{ País/idioma } & \multirow[b]{2}{*}{ Genero } & \multirow[b]{2}{*}{$\begin{array}{c}\text { Edad } \\
\text { años }\end{array}$} & \multicolumn{5}{|c|}{ Indicadores de bondad de ajuste } \\
\hline & & & CFI & TLI & $\begin{array}{c}\text { RMS } \\
\text { EA }\end{array}$ & $\begin{array}{c}\alpha \\
\text { Cronbach }\end{array}$ & $\mathbf{x}^{2}$ valor $p$ \\
\hline \multicolumn{8}{|l|}{$\begin{array}{c}\text { ORTO-15 } \\
\text { (3 dominios) }\end{array}$} \\
\hline Alemania/alemán & Femenino y Masculino & $19-70$ & 0,78 & 0,74 & 0,07 & 0,30 & $466,4(0,001)$ \\
\hline Hungría/húngaro & Femenino y Masculino & $20-70$ & 0,72 & 0,66 & 0,11 & & $931,2(0,001)$ \\
\hline Perú/español & Femenino & $18-30$ & 0,79 & 0,74 & 0,07 & 0,73 & $204,8(0,001)$ \\
\hline \multicolumn{8}{|l|}{$\begin{array}{c}\text { ORTO-11 } \\
\text { (1 dominio) }\end{array}$} \\
\hline Hungría/húngaro & Femenino y Masculino & $20-70$ & 0,92 & 0,90 & 0,08 & 0,82 & \\
\hline Perú/español & Femenino & $18-30$ & 0,85 & 0,82 & 0,07 & 0,74 & $102,9(0,001)$ \\
\hline \multicolumn{8}{|l|}{$\begin{array}{c}\text { ORTO-9 } \\
\text { (1 dominio) }\end{array}$} \\
\hline Alemania/alemán & Femenino y Masculino & $19-70$ & 0,95 & 0,92 & 0,05 & 0,67 & $83,8(0,001)$ \\
\hline Perú/español & Femenino & $18-30$ & 0,75 & 0,67 & 0,10 & 0,64 & $91,3(0,010)$ \\
\hline
\end{tabular}

CFI: índice de ajuste comparativo, TLI: índice de Tucker-Lewis, RMSEA: raíz del residuo promedio de proximación, $\mathrm{x}^{2}$ : estadístico ji cuadrado. 


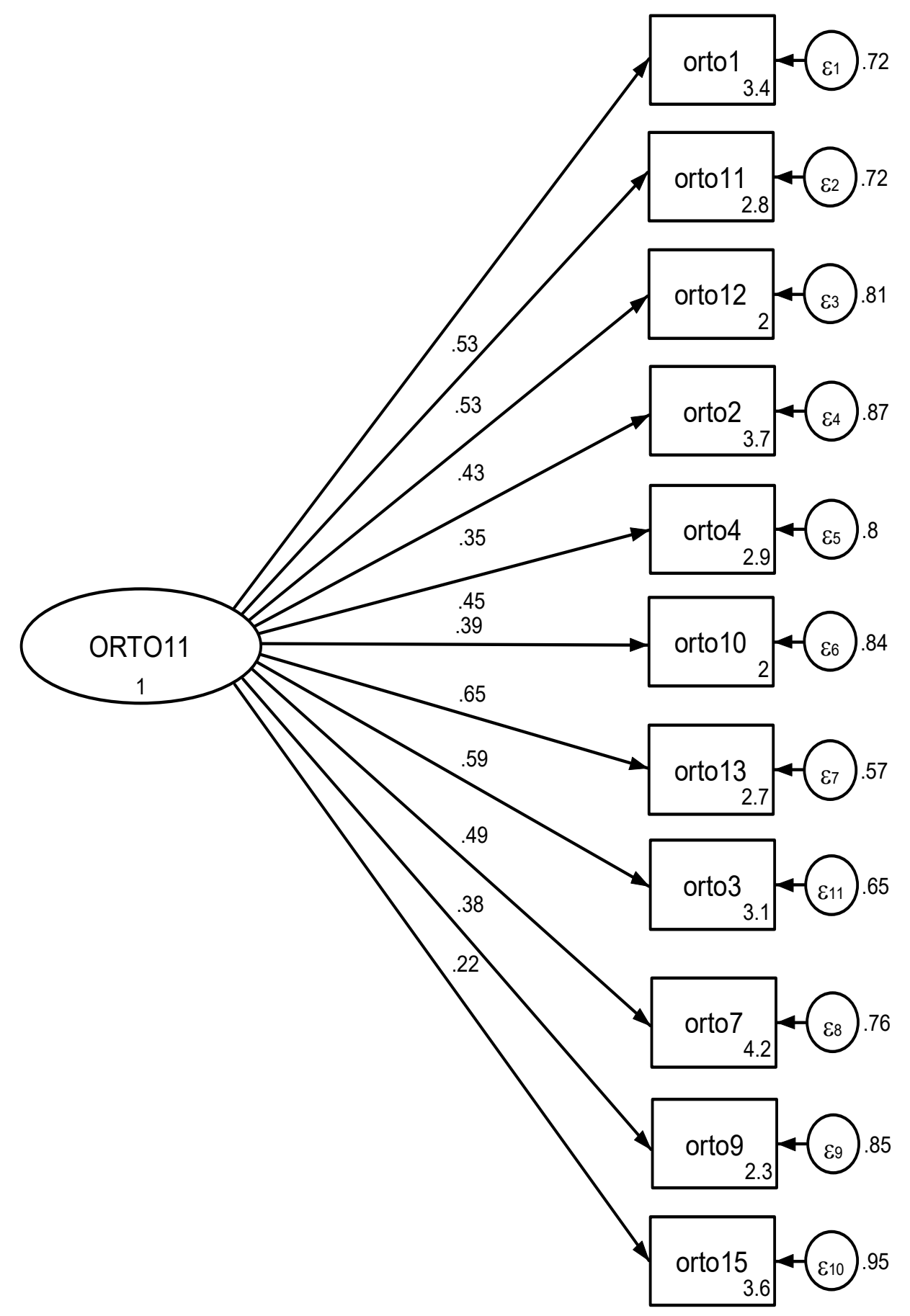

Figura 2. Mejor modelo final de ajuste ORTO 11 con una dimensión. 
Tabla 3. Comparación entre el factor de carga estandarizado del ORTO 15 y ORTO 11.

\begin{tabular}{|c|c|c|c|c|}
\hline \multirow[b]{2}{*}{ ÍTEMS } & \multicolumn{2}{|l|}{$\begin{array}{l}\text { ORTO } 15 \\
3 \text { Factores }\end{array}$} & \multicolumn{2}{|l|}{$\begin{array}{l}\text { ORTO } 11 \\
1 \text { Factor }\end{array}$} \\
\hline & $\begin{array}{l}\text { Factor de } \\
\text { carga } \\
\text { estandarizado }\end{array}$ & $\begin{array}{cc}\text { Varianza no } \\
\text { explicada }\end{array}$ & $\begin{array}{l}\text { Factor de } \\
\text { carga } \\
\text { estandarizado }\end{array}$ & $\begin{array}{r}\text { Varianza no } \\
\text { explicada }\end{array}$ \\
\hline Orto1 & 0,57 & 0,67 & 0,53 & 0,72 \\
\hline Orto2 & 0,29 & 0,92 & 0,35 & 0,87 \\
\hline Orto3 & 0,63 & 0,60 & 0,59 & 0,65 \\
\hline Orto4 & 0,43 & 0,82 & 0,45 & 0,80 \\
\hline Orto5 & $-0,12$ & 0,99 & - & - \\
\hline Orto6 & 0,44 & 0,81 & - & - \\
\hline Orto 7 & 0,56 & 0,68 & 0,49 & 0,76 \\
\hline Orto8 & 0,17 & 0,97 & - & - \\
\hline Orto9 & 0,39 & 0,85 & 0,38 & 0,85 \\
\hline Orto10 & 0,41 & 0,84 & 0,39 & 0,84 \\
\hline Orto11 & 0,50 & 0,75 & 0,53 & 0,72 \\
\hline Orto12 & 0,54 & 0,71 & 0,43 & 0,81 \\
\hline Orto13 & 0,55 & 0,70 & 0,65 & 0,57 \\
\hline Orto14 & 0,30 & 0,91 & - & - \\
\hline Orto15 & 0,22 & 0,95 & 0,22 & 0,95 \\
\hline
\end{tabular}

\section{Fiabilidad}

Se encontró una satisfactoria consistencia interna del ORTO-11 con un alfa de Cronbach de 0,74. A nivel de ítems se identificó que ninguno tuvo una variación significativa entre el pre y post test ( $\mathrm{p}>0,20$; prueba de los signos de Wilcoxon). No se encontró diferencia de puntajes global del ORTO-11 en el pre y post test $(\mathrm{p}=0,35$; prueba de los signos de Wilcoxon) con una correlación intraclase buena $(\mathrm{CCI}=0,70)$.

\section{Asociación entre el puntaje del ORTO-11 y otras variables}

El promedio del puntaje del cuestionario ORTO-11, fue 27,9 para Nutrición y Dietética y 28,0 para Diseño de Interiores. No se encontró diferencias según carrera, edad, nivel socioeconómico medido a partir de la pensión universitaria, antecedentes familiares e índice de masa corporal (IMC). Aquellas estudiantes que practicaban algún deporte, o específicamente aeróbicos o pesas, tuvieron puntajes más bajos (mayor tendencia de $\mathrm{ON}$ ) en el ORTO-11 ( $<<0,05$, Tabla 4). 
Tabla 4. Relación entre el puntaje de ORTO 11 y las características socio-demográficas y conductuales $(\mathrm{N}=258)$.

\begin{tabular}{|c|c|c|c|c|}
\hline \multirow[t]{2}{*}{ Características } & \multicolumn{3}{|c|}{ Puntaje de ORTO 11} & \multirow[t]{2}{*}{ Valor $\mathbf{p}$} \\
\hline & $\mathbf{N}$ & Media & DS & \\
\hline Edad & $19,9 *$ & 0,08 & $* *$ & 0,191 \\
\hline \multicolumn{5}{|l|}{ Facultad } \\
\hline Nutrición y dietética & 108 & 27,9 & $(3,3)$ & 0,811 \\
\hline Diseño de interiores & 150 & 28,0 & $(2,9)$ & \\
\hline \multicolumn{5}{|l|}{ Pensión } \\
\hline$<1900$ & 203 & 28,1 & $(3,0)$ & 0,090 \\
\hline$>=1900$ & 55 & 27,3 & $(3,1)$ & \\
\hline \multicolumn{5}{|l|}{ Antecedente familiar } \\
\hline Sí & 72 & 27,9 & $(3,1)$ & 0,933 \\
\hline No & 186 & 27,9 & $(3,0)$ & \\
\hline $\begin{array}{l}\text { Practica de algún } \\
\text { deporte }\end{array}$ & & & & \\
\hline $\mathrm{Si}$ & 147 & 27,3 & $(2,9)$ & 0,002 \\
\hline No & 111 & 28,4 & $(3,0)$ & \\
\hline IMC & & & & 0,323 \\
\hline$<18,5$ & 13 & 29,1 & $(2,5)$ & \\
\hline $18,5-24,9$ & 194 & 27,9 & $(3,0)$ & \\
\hline $25,0-<30,0$ & 51 & 27,8 & $(3,3)$ & \\
\hline
\end{tabular}

DS: desviación estándar. * Promedio de edad, **Coeficiente de correlación de Pearson. 


\section{DISCUSIÓN}

Nuestro estudio realizó la traducción y validación del instrumento ORTO-15 (versión original italiana) que mide la tendencia de ON, al idioma español, se analizó sus propiedades psicométricas. Considerando los resultados de AFC y consistencia interna, se encontró que el ORTO-11 fue el instrumento que mejor se adaptó a la realidad de nuestro país. Este cuestionario evalúa un solo factor y omite las preguntas 5, 6, 8 y 14. De manera similar, Arusoglu et al proponen una variación al ORTO- 15 en la cual omiten también cuatro preguntas, pero distintas a las omitidas en el presente estudio $(1,2,9,15)$. (8) Sin embargo, el instrumento evaluado en el estudio de Varga et al fue el que presentó una mejor consistencia interna que el de Arusoglu et al. (9)

En el presente estudio, creemos que los ítems que fueron retirados no afectan los criterios que definen la tendencia a la ON. Los criterios excluidos se basan en el sabor de los alimentos, la disposición en el gasto de alimentos saludables, el rechazo a algún grupo de alimentos y su percepción sobre los puntos de adquisición de los alimentos no saludables. Por el contrario, por Arusoglu et al sí creemos que omiten las preguntas que definen las características de las tendencias ortoréxicas. Como por ejemplo el pensar la cantidad del consumo diario de calorías, el consumo de alimentos a solas, dificultad a la hora de elegir los alimentos. Siendo las características de la tendencia a ON más resaltantes, los sentimientos de culpabilidad, la personalidad controladora y perfeccionista, el aislamiento social, el omitir alimentos con aditivos, el utilizar utensilios especiales para la preparación de los alimentos, la dedicación obsesiva a la preparación de sus alimentos, la preocupación por la calidad más no por la cantidad, entre otras. (17) Por ello, los ítems seleccionados para el ORTO-11 podrían ser de buena utilidad para medir la tendencia a ON.

Se evaluó la correlación entre el puntaje total obtenido del ORTO-11 con el EAT-26, hallándose un coeficiente de correlación de Pearson de $-0,44(p<0,001)$. Asimismo, Stochel et al, también analizan la correlación entre los cuestionarios ORTO-15 y EAT-26, reportando una relación entre los hábitos de alimentación anormales y las preocupaciones 
sobre el peso corporal con un coeficiente de correlación entre los dos factores de $-0,12$ $(p=0,01)$. (5) El EAT-26 es una prueba de tamiz que mide un conjunto de actitudes y conductas asociadas con los trastornos alimentarios conductuales (18), los cuales como se puede observar se asocian al cuestionario ORTO-11. El presente estudio obtuvo una mejor correlación en comparación al estudio de Stochel et al.

Nuestro instrumento presentó un alfa de Cronbach de 0,74 , muy parecido al obtenido por Stochel et al en una población de jóvenes de 15 a 21 años utilizando el cuestionario ORTO15 (0,78). (6) Podemos decir que el ORTO-11 obtuvo una buena consistencia interna. Lo que afirma que es un cuestionario confiable y sirve para evaluar las actitudes obsesivas relacionadas a la nutrición. (19) Por lo tanto, podemos decir que para la población femenina el ORTO-11 podría ser de buena utilidad.

No se encontró asociación significativa de la tendencia a $\mathrm{ON}$ entre las carreras de Nutrición y Dietética y Diseño de Interiores. Un estudio realizado en Alemania por Korinth et al también comparó la tendencia de ON en estudiantes de Nutrición y otras carreras, donde tampoco se encontró diferencias significativas; sin embargo, reportó una menor tendencia de padecer ON en estudiantes de Nutrición de años avanzados. (20) Bağci-Bosi et al halló en Turquía que el 40,9\% de nutricionistas presentaban riesgo de $\mathrm{ON}$ en ambos géneros y en Brasil se encontró que el $88,7 \%$ de los estudiantes de Nutrición presentaron tendencia de ON, donde el 75\% tenía una distorsión de la imagen corporal. (21-22) Asimismo, un estudio mostró que los profesionales de la salud como las nutricionistas y médicos, tienen mayor riesgo de $\mathrm{ON}$, debido a que como profesionales tienen como objetivo principal trasmitir estilos de vida saludables pudiéndose tornar en un hábito obsesivo. (7) Además, un 43,6\% de médicos presentó comportamientos con características altamente sensibles a la ON teniendo un porcentaje similar al de un estudio realizado en Turquía con nutricionistas.

Se encontró mayor puntaje de ON en mujeres que realizan deporte, siendo mayor en casos donde el tipo de deporte es aeróbico. Asimismo, en Italia se realizó un estudio por SeguraGarcía et al donde se observó una alta tendencia de ON en mujeres $(28 \%)$ y hombres $(30 \%)$ 
atletas. (24) Por ello se puede considerar que los atletas pueden ser considerados un grupo de riesgo, ya que tienden a controlar su alimentación siendo para ellos crucial obtener un buen rendimiento físico.

En el presente estudio, no se evaluó la validez convergente con otras características vinculadas al concepto de $\mathrm{ON}$ como lo hicieron con el consumo de alimentos saludables.

Finalmente, la selección de la población fue exclusivamente estudiantes universitarios de género femenino, por lo tanto no podemos extrapolar a la población general.

Si bien hemos validado el ORTO-11 para su uso en español, queda pendiente evaluar si puede discriminar entre personas con diagnóstico clínico de $\mathrm{ON}$, identificar su punto de corte y sensibilidad y especificidad. Por el momento, puede usarse como una escala que da intensidad de síntomas, donde a mayor puntaje menor sintomatología relacionada con ON. 


\section{CONCLUSIONES}

La adaptación propuesta del ORTO 11 validada al español presentó mejores propiedades psicométricas que la del cuestionario ORTO15. Finalmente, recomendamos establecer un punto de corte que permita evaluar la tendencia a ON usando la nueva versión ORTO 11 en futuros estudios. 


\section{REFERENCIAS BIBLIOGRÁFICAS}

(1) Bratman S, Knight D. Orthorexia nervosa: overcoming the obsession with healthful eating. Health food junkies. Nueva York: Broadway Books; 2000. 242p.

(2) Martins MC, Alvarenga M, Vargas S, Santo K, Scagliusi F. Ortorexia nervosa: reflexões sobre um novo conceito. Revista de nutricao. 2011; 24(2):345-57.

(3) Donini LM, Marsili D, Graziani MP, Imbriale M, Cannella C. Orthorexia Nervosa: Validation of a diagnosis questionary. Eat Weight Disorder. 2005;10(2); 28-32.

(4) American Psychiatric Association Diagnostic and Statistical Manual of Mental Disorders. 5th ed. Washington: American Psychiatric Association; 2013.

(5) Brytek-Matera A, Donini L, Krupa M, Poggiogalle E, Hay P. Orthorexia nervosa and self-attitudinal aspects of body image in female and male university students. J Eat Disorders. 2015;3:2.

(6) Stochel M, Janas-Kozik M, Zejda J, Hyrnik J, Jelonek I, Siwiec A. Validation of ortho 15 questionnaire in the group of urban youth aged 15-21. Psychiatr Pol. 2015;49(16):119-34.

(7) Bağci-Bosi AT, Camur D, Güler C. Prevalence of orthorexia nervosa in resident medical doctors in the faculty of medicine (Ankara, Turkey). Appetite. 2007;49(3):661-6.

(8) Arusoglu G, Kabakci E, Koksal G, Merdol T. ORTO 11 Orthorexia nervosa and adaptation of Orto 11 into turkish. Turk Jour Psy 2008;19(3):283-91.

(9) Varga M, Thege BK, Dukay-Szabó S, Túry F, van Furth EF. When eating healthy is not healthy: orthorexia nervosa and its measurement with the ORTO15 in Hungary. BMC Psychiatry 2014;14:59.

(10) Missbach B, Hinterbuchinger B, Dreiseitl V, Zellhofer S, Kurz C, Konig J. When eating right, is measured wrong! A validation and critical examination of the ORTO 15 Questionnaire in German. PLOS ONE 2015;10(8): e0135772.

(11) Cavero V, Lodwig C, Lulli G, Tejada R. A new challenge for health care professionals. Rev Alan. 2015; 65(3). 
(12) Muñiz J, Elosua P, Hambleton R. Directrices para la traducción y adaptación de los test: segunda edición. Psicoterma 2013; 25(2):151-7.

(13) Varela-Ruiz M, Díaz-Bravo L, Garcia-Duran K. Descripción y usos del método Delphi en investigaciones del área de salud. Inv Ed Med 2012;1(2):90-95.

(14) Constain G, Ricardo C, Rodríguez-Gázquez M, Álvarez M, Marín C, Agudelo C. Validez y utilidad diagnostica de la escala EAT-26 para la evaluación del riesgo de trastornos de la conducta alimentaria en población femenina de Medellín, Colombia. Aten Primaria. 2014;46(6):283-89.

(15) Costello A, Osborne J. Best practices in exploratory factor analysis: Four recommendations for getting the most from your analysis. PARE. 2005;10(7):1-9.

(16) Aguilar L, Contreras M, Del Canto J, Vilchez W. Guía técnica para la valoración nutricional antropométrica de la persona adulta. MINSA. 2012.

(17) Koven NS, Abry AW. The clinical basis of orthorexia nervosa: emerging perspectives. Neuropsychiatr Dis Treat. 2015;11:385-94.

(18) Harris N, Gee D, D Acquisto D, Ogan D, Pritchett K. Eating disorder risk, exercise dependence, and body weight dissatisfaction among female nutrition and exercise science university majors. J Behav Addict. 2015;4(3): 206-9.

(19) Brytek-Matera A, Krupa M, Poggiogalle E, Donini M. Adaptation of the ORTHO-15 test to polish women and men. Eat Weight Disorders. 2014;19(1):69-76.

(20) Korinth A, Schiess S, Westenhoefer J. Eating behaviour and eating disorders in students of nutrition sciences. Public Health Nutr. 2010;13(1):32-7.

(21) Asil E, Sürücüoğlu MS. Orthorexia nervosa in Turkish dietitians. Ecol Food Nutr. 2015;54(4):303-313.

(22) OctacilioQ, Rodrigues A. Comportamento de risco para ortorexia nervosa em estudantes de nutrição. J Bras Psiquiatr. 2014;63(3):200-4. 
(23) Segura-García C, Papaianni MC, Caglioti F, Procopio L, Nisticò CG, Bombardiere L, Ammendolia A, Rizza P, De Fazio P, Capranica L. Orthorexia nervosa: a frequent eating disordered behavior in athletes. Eat Weight Disord. 2012;17(4):226-33. 


\title{
ANEXOS
}

\section{ANEXO 1: ACTA DE SUSTENTACIÓN}

\author{
(U) UPC \\ Univirsidad Peruna di Ciencins Arugads
}

\section{ACTA DE SUSTENTACIÓN}

En el día de hoy se reúne el jurado integrado por:

\begin{tabular}{|l|l|}
\hline Presidente & Sandra Cusirramos Jiménez \\
\hline Jurado & Eddy Segura Paucar \\
\hline Jurado & Mario Reyes Bossio \\
\hline
\end{tabular}

para evaluar la sustentación de:

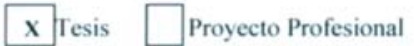

Expedientes

titulado: TRADUCCIÓN Y VALIDACIÓN DE LA VERSIÓN AL ESPAÑOL DEL CUESTIONARIO ORTO-15 PARA LA EVALUACIÓN DE ORTOREXIA

desarrollado por:

Vanessa Gabriela Cavero Amayo

Cynthia Lodwig León

Gabriela Lulli Rodriguez-Larrain

asesorado por:

Romina Tejada Caminiti

Jorge Maguiña Quispe

para optar por el título

profesional de:

Licenciada en Nutrición y Dietética

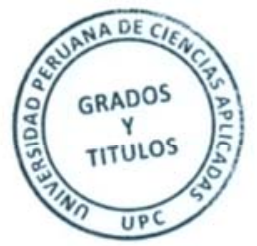

Después de haber escuchado la exposición, así como las respuestas a las preguntas formuladas en la defensa, el jurado concluye que el/los graduado(s) ha(n) demostrado estar preparado(s) para iniciar el ejercicio profesional. Por lo tanto, teniendo en cuenta los rangos de calificación siguiente:

/ Aprobado / Notable / Sobresaliente / Summa Cum Laude / Desaprobado /

el jurado otorga el siguiente resultado a:

\begin{tabular}{|l|l|}
\hline \multicolumn{1}{|c|}{ Estudiante } & Calificación \\
\hline Vanessa Gabriela Cavero Amayo & Notable \\
\hline Cynthia Lodwig León & Notable \\
\hline Gabriela Lulli Rodriguez-Larrain & Notable \\
\hline
\end{tabular}

Dado en la ciudad de Lima a los 09 dias del mes de giciembre de 2016.
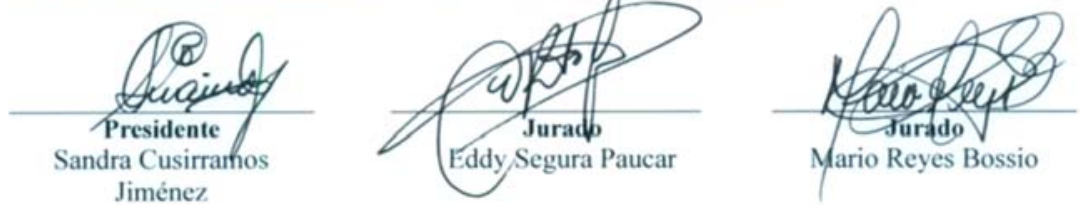


\section{ANEXO 2: APROBACIÓN DEL COMITÉ DE ÉTICA}
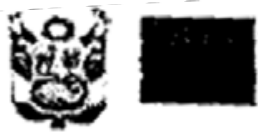

Ministerio de

Salud

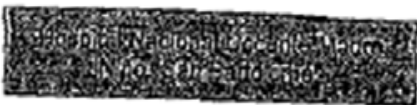

DIRECCION DE APOYO A LA

DOCENCIA E INVESTIGACIÓN

Lima, 10 de marzo del 2015

\section{OFICIO No 109-OADI-HONADOMANI.SB-2015}

Setiotita

VANESSA CAVERO

Estudiante de la Facultad de Ciencias de la Salud

Universidad Peruana de Ciencias Aplicadas

Presente.-

Expediente $\mathrm{N}^{\circ} 02777-15$

Tenemos el agrado de saludar a usted, con la finalidad de responder al documento de la referencia, sobre el pedido de revisión y aprobación del estudio de Investigación titulado: "RIESGO DE ORTOREXIA EN ESTUDIANTES DE UNA UNTVERSIDAD PRIVADA DE LIMA".

Los Comités de Etica Institucional y Investigación, manifiestan que en reunión ordinária Hevado a cabo en la Oficina de de Apoyo a la Docencia e Investigación, dicho ESTUDIO DE INVESTIGACION HÁ SIDO APROBADO.

REVISOR: Dr. Nazario Silva Astete.

Nos es propicia la oportunidad para renovar los sentimientos de nuestra mayor consideración.

Atentamente,
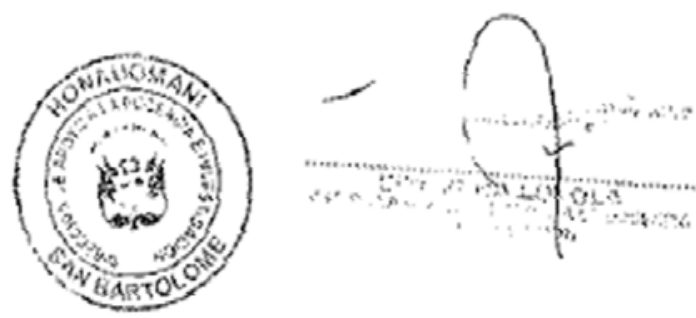


\section{ANEXO 3: INSTRUMENTO DE RECOLECCIÓN DE DATOS}

\section{Consentimiento Informado para participar en un estudio de investigación}

Institución: Universidad Peruana de Ciencias Aplicadas

Investigadores: Cynthia Lodwig, Vanessa Cavero y Gabriela Lulli

Título: Riesgo de ortorexia en estudiantes de una universidad privada de Lima.

Propósito del estudio: Le invitamos a participar de un estudio llamado Riesgo de ortorexia en estudiantes de una universidad privada de Lima. Entiéndase como ortorexia a la obsesión por la alimentación saludable. Este estudio será desarrollado por alumnas de pregrado de la carrera de nutrición y dietética para optar el título profesional de licenciada en nutrición. La importancia de este estudio está en identificar si la frecuencia de estudiantes del sexo femenino con ortorexia y saber si hay mayor incidencia en los estudiantes de la carrera de nutrición y dietética en comparación con la carrera de diseño de interiores.

Procedimientos a realizar:

Si usted accede a participar en este estudio, formará parte de los siguientes procedimientos:

1. Usted recibirá un cuestionario autoaplicativo que consta de 25 preguntas que incluyen preguntas referidas a medir la ortorexia que consta de 15 preguntas, datos sociodemográficos, económicos, actividad física y antecedentes patológicos que constan de 9 preguntas.

2. Luego de completar dicho instrumento usted será pesado y tallado y se le calculará el índice de masa corporal.

Todo el procedimiento descrito anteriormente mencionado tendrá una duración de 10 minutos.

Riesgos: No se han previsto riesgos por participar en esta fase del estudio. La toma del peso y la talla podría generarle cierta incomodidad, sin embargo estos procedimientos serán realizados por personas capacitadas con el más mínimo cuidado y máximo respeto por su persona.

Beneficios: Se beneficiará por tener una evaluación nutricional que consta del peso y la talla, además del cálculo de su índice de masa corporal. También recibirá el resultado del test de ortorexia si usted lo requiere.

Fuentes de financiamiento, costos e incentivos: Usted no deberá pagar nada por participar en el estudio, igualmente, no recibirá ningún incentivo económico ni de otra índole, únicamente la satisfacción de participar y colaborar en el estudio.

Confidencialidad: Se le asignará un código al momento de recibir el cuestionario, de la misma manera en la base de datos donde se registrará su peso y su talla también contará con su código asignado. Si los resultados de este estudio son publicados, no se revelará 
información alguna que permita la identificación de las personas que participaron. Sus resultados no serán mostrados a ninguna persona ajena al estudio sin su consentimiento.

Uso futuro de la información obtenida: Los resultados obtenidos serán utilizados para la publicación de un estudio de investigación, que nos permita optar por el título de Licenciado en Nutrición y Dietética.

Derechos del participante: Si usted decide participar en el estudio, puede retirarse de éste en cualquier momento, o no participar en una parte del estudio sin perjuicio alguno. Si tiene alguna duda adicional, por favor consúltelo con las investigadoras del estudio. Una vez culminado el estudio, los investigadores se comunicarán con usted para poder enviarle los resultados de los exámenes realizados. Si tiene alguna inquietud no dude en escribirnos al correo electrónico: cynlod@hotmail.com, dirigido a Cynthia Lodwig León. Cualquier consulta sobre el estudio y los procedimientos del mismo las podrá hacer a las investigadoras: Vanessa Cavero cuyo email es vcavero93@hotmail.com y Gabriela Lulli cuyo emailgabrielalrl@hotmail.com.

En caso usted se sienta vulnerado, puede contactarse con el Comité de Ética e investigación de la Universidad Peruana de Ciencias Aplicadas a través de la Sra. Carla Lira al teléfono 3133333 anexo 2701 o al correo electrónico carla.lira@upc.edu.pe

\section{CONSENTIMIENTO}

Aplicado por:

Acepto voluntariamente participar en este estudio, comprendo cual es la finalidad del proyecto, para qué y cómo serán utilizados mis datos, también entiendo que puedo decidir no participar y que puedo retirarme del estudio en el momento que yo desee.

Participante

Nombre:

Fecha:

Firma:

Investigadora

Nombre:

Fecha:

Firma: 
Indicaciones: Por favor, marcar con una $\mathrm{X}$ dentro del recuadro y rellenar donde se especifique.

¿Qué edad tienes? años

Facultad 0. Nutrición

__ 1. Diseño de interiores

Pensión universitaria $\underline{\text { (Sin contar la beca) }}$

$\mid \quad 0 .<1459$ soles

2. 1900-2199soles

4. $>2500$ soles

1. 1500-1899 soles

3. 2200-2500 soles

Nro de cuotas

Marca con una $X$ aquellas enfermedades que han padecido tus FAMILIARES en primer grado (padres, hermanos, hijos) (puede marcar más de una opción)

$\mid \_$0. Dislipidemias (colesterol y triglicéridos altos) $\left|\_\right| 2$. Diabetes $\quad\left|\_\right|$4. Alergias alimentarias
|1. Cáncer
|3. Hipertensión
|5.Ninguna

Otros Especifique

Marca con una X si padeces de alguna de estas enfermedades (puede marcar más de una opción):

L_ 0. Dislipidemias (colesterol y triglicéridos altos) $\left|\_\right|$2. Diabetes $\quad$ L_4. Alergias alimentarias $\mid$ L 1 . Cáncer |3. Hipertensión |5. Ninguna

Otros Especifique

¿Realizas deporte? (Respuesta NO, pasar a antropometría) $\left|\_\right| 0$.No

|1. Si

Número de días a la semana _ _ días

Cantidad de horas por día _ Horas

¿Qué deporte realizas? (Puedes marcar más de una opción)

L_|0. Futbol L_|3. Yoga/ Pilates $\quad$ _ $\mid 6$. Aeróbicos (correr, spinning)

L 1 1.Artes marciales

4. Atletismo

17. Pesas

L_|2. Tenis/squash/frontón

5. Natación

8. Otros especificar

\section{Antropometría (DATOS TOMADOS POR EL ENCUESTADOR)}

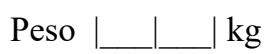

Talla

$\mathrm{m}$

IMC $\mathrm{Kg} / \mathrm{m}^{2}$

PASE A LA SIGUIENTE

PÁrINA 


\begin{tabular}{|c|c|c|c|c|}
\hline Orto 15 & Siempre & $\begin{array}{l}\text { Casi } \\
\text { siempre }\end{array}$ & A veces & Nunca \\
\hline $\begin{array}{l}\text { ¿Piensas en cuántas calorías consumes cuando } \\
\text { comes? }\end{array}$ & & & & \\
\hline $\begin{array}{l}\text { ¿En una tienda de productos alimentarias es difícil } \\
\text { para ti elegir alimentos? }\end{array}$ & & & & \\
\hline $\begin{array}{l}\text { ¿En los últimos tres meses pensar en comida ha sido } \\
\text { una preocupación para ti? }\end{array}$ & & & & \\
\hline $\begin{array}{l}\text { ¿Lo que eliges para comer está condicionado por la } \\
\text { preocupación por tu estado de salud? }\end{array}$ & & & & \\
\hline $\begin{array}{l}\text { ¿El sabor es la cualidad más importante que tiene en } \\
\text { cuenta a la hora de juzgar un alimento? }\end{array}$ & & & & \\
\hline $\begin{array}{l}\text { ¿Estás dispuesta gastar más dinero por una } \\
\text { alimentación saludable? }\end{array}$ & & & & \\
\hline $\begin{array}{l}\text { ¿El pensar en comida sana ha sido una preocupación } \\
\text { por más de } 3 \text { horas? }\end{array}$ & & & & \\
\hline $\begin{array}{l}\text { ¿Tienes algún rechazo o negación a un alimento o } \\
\text { grupo de alimentos? }\end{array}$ & & & & \\
\hline $\begin{array}{l}\text { ¿Consideras que el estado de ánimo afecta cómo y } \\
\text { cuánto decido comer? }\end{array}$ & & & & \\
\hline $\begin{array}{l}\text { ¿Crees que sea verdad que la comida sana te hace } \\
\text { sentir bien contigo misma? }\end{array}$ & & & & \\
\hline $\begin{array}{l}\text { ¿Consideras que el consumo de comida sana } \\
\text { modifica tu comportamiento con tus amistades } y / o \\
\text { familiares }\end{array}$ & & & & \\
\hline $\begin{array}{l}\text { ¿Consideras que consumir comida sana puede } \\
\text { mejorar tu aspecto físico? }\end{array}$ & & & & \\
\hline $\begin{array}{l}\text { ¿Te sientes culpable cuando consumes alimentos } \\
\text { poco saludables? }\end{array}$ & & & & \\
\hline $\begin{array}{l}\text { ¿Consideras que el mercado actual de productos } \\
\text { alimentarios tiene en su mayoría productos no } \\
\text { sanos? }\end{array}$ & & & & \\
\hline $\begin{array}{l}\text { ¿Actualmente cuando consumes tus alimentos lo } \\
\text { haces solo? }\end{array}$ & & & & \\
\hline
\end{tabular}




\section{ANEXO 4: DIAGRAMA DE FLUJO DE LOS PROCESOS REALIZADOS EN EL PRESENTE ESTUDIO}

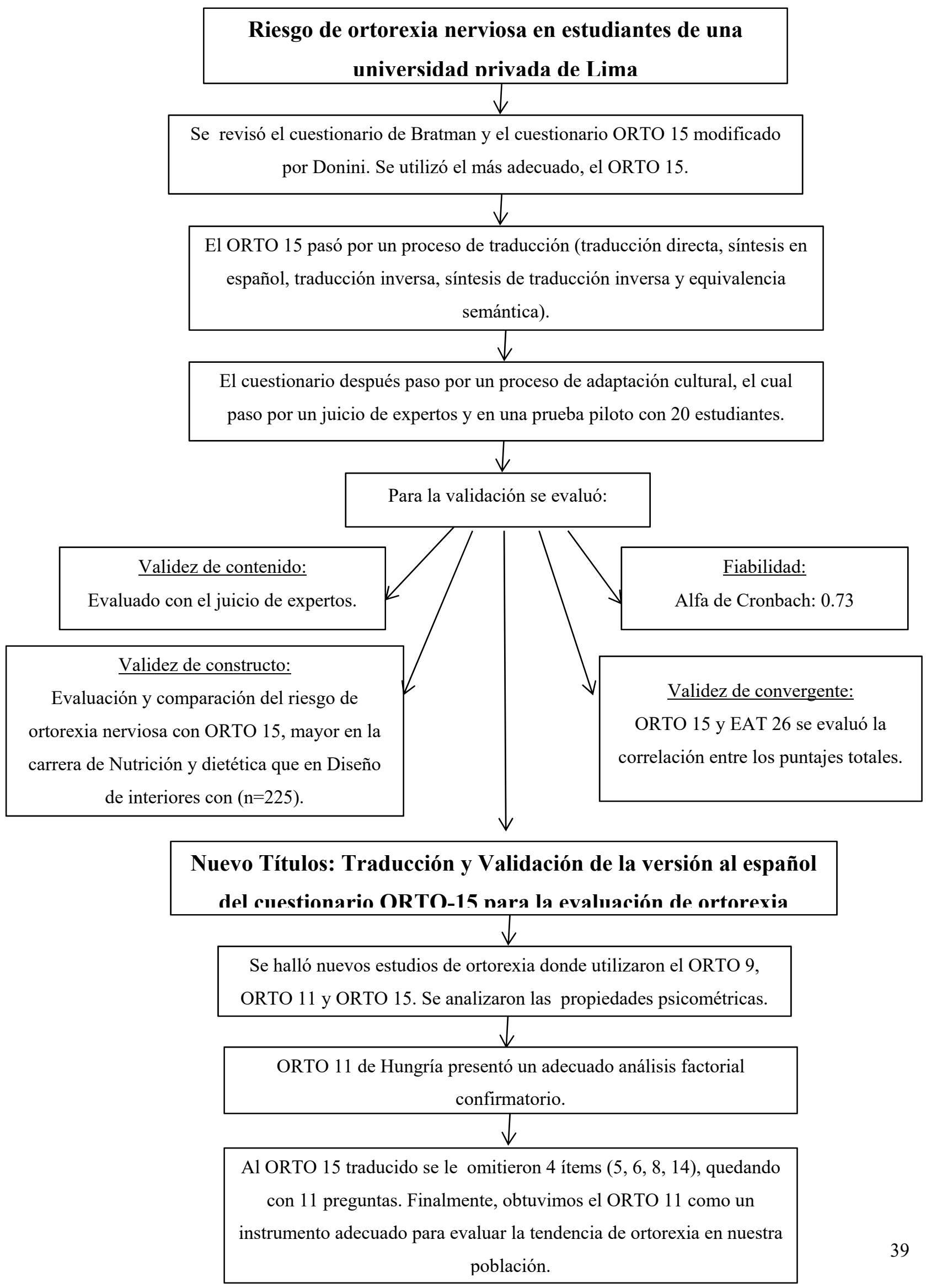

